\title{
A Abstração Reflexionante no Pensamento Computacional e no Desenvolvimento de Projetos de Robótica em um Makerspace Educacional
}

\author{
Maria Inês Castilho, PPGIE-UFRGS, minescastilho@gmail.com \\ Karen Selbach Borges, PPGIE-UFRGS e IFRS, karen.borges@poa.ifrs.edu.br \\ Léa da Cruz Fagundes, UFRGS, leafagundes@gmail.com
}

Resumo: O pensamento computacional tem sido tema recorrente nas últimas décadas e preconiza a abstração como base estrutural do processo. Considerando a teoria cognitiva de Jean Piaget, a robótica educacional e a cultura maker, buscamos demonstrar que o desenvolvimento das habilidades do pensamento computacional está centrado na abstração reflexionante. Para a efetivação das atividades de pesquisa foi oportunizado um Curso de Robótica para Adolescentes, no laboratório de fabricação digital - POALab FabLab - dirigido a alunos do $1^{\circ}$ ano do ensino médio de escolas públicas. A capacidade de abstração dos participantes foi continuamente estimulada através de perguntas dirigidas à reflexão enquanto realizavam atividades de robótica, ou de uso das máquinas do laboratório, que demandavam a aplicação do pensamento computacional. Concluímos, a partir desse estudo, que a abstração reflexionante está presente no desenvolvimento de todas as habilidades do pensamento computacional, sendo adequado se utilizar da robótica como meio para seu desenvolvimento.

Palavras-chave: pensamento computacional, robótica educacional, cultura maker, abstração reflexionante

\begin{abstract}
The computational thinking has been widely discussed in the last decade and is common sense that the abstraction is one of the most important characteristics. Considering Jean Piaget's cognitive theory, educational robotics and the maker culture, we intend to demonstrate that computational thinking skills demand reflective abstraction. To verify this hypothesis, we conducted an Educational Robotics Workshop for students enrolled in the first year of high school. The activities took place at a digital fabrication laboratory - POALab FabLab. The use of abstraction were continually stimulated by reflective questions, asked while the students applied computational thinking to build their robots using digital fabrication machines. As a result, we concluded that reflective abstraction is necessary to develop the computational thinking skills and the educational robotics activities are suitable for this.
\end{abstract}

Key-words: computational thinking, educational robotics, maker culture, reflective abstraction. 


\section{Introdução}

A capacidade de formular e resolver problemas são qualidades que todos desejam possuir. Identificar um problema é diferente de formular um problema, porque essa segunda situação dá margem a possíveis soluções, ou seja, a levantamento de hipóteses, que poderão ser testadas e verificadas. Para resolver problemas em qualquer área, nas últimas décadas, tem se abordado o desenvolvimento das habilidades características do Pensamento Computacional (PC). Quando Jeannete Wing (2006), afirmou que o PC envolve habilidades de resolução de problemas, baseando-se nos conceitos fundamentais da ciência da computação, estava abrindo espaço para discussão e aplicação desse processo em qualquer situação, independente de uso de máquinas digitais ou não.

Aho e Ullman (1992), afirmam que o pensamento computacional "é uma ciência da abstração” e como tal, essa forma de pensamento se consolida. Segundo Wing (2014), a abstração aplicada pelo pensamento computacional nos dá o poder de dimensionar e lidar com a complexidade. Nesse sentido, queremos verificar se a robótica educacional desenvolvida num espaço maker, oportuniza abstração reflexionante. Ao mesmo tempo, procuramos identificar se ocorrem abstrações reflexionantes durante a aplicação de cada habilidade do pensamento computacional.

Assim, organizamos este artigo de modo a oferecer, através da seção 2, uma visão geral sobre a cultura maker e a robótica educacional. Nas duas seções seguintes apresentaremos o conceito de abstração reflexionante, relacionando-a com o conceito de pensamento computacional. Nas seções 5 e 6, respectivamente, iremos detalhar o método de trabalho adotado para essa pesquisa e os dados coletados, analisando-os a partir da teoria de Jean Piaget. Conclusões e indicações de trabalhos futuros encontramse na seção 7.

\section{Cultura Maker e Robótica Educacional}

A cultura maker é o produto do casamento entre a cultura hacker e o movimento DIY (Do it yourself ou faça você mesmo). A cultura hacker é a favor do software livre e do hardware aberto e livre, o que tornou possível a existência de sistemas operacionais de código aberto, como o Linux, além de plataformas de hardware como o Arduino. $\mathrm{O}$ movimento DIY, por sua vez, procura difundir a ideia de que é possível construir, modificar ou consertar coisas por conta própria, sem ter de recorrer à indústria ou a profissionais caros. Essa ideia do "faça você mesmo", usando software e hardware livres foi um impulso para os métodos ativos de aprendizagem como, por exemplo, a aprendizagem baseada em projetos de robótica educacional.

Entretanto, antes mesmo de se falar em cultura maker, a robótica já estava presente no meio educacional graças ao projeto Logo de Seymour Papert e os kits com blocos de montar da Lego. O que mudou de lá para cá é que hoje existe uma grande diversidade de kits robóticos a disposição, além da possibilidade de, graças às plataformas de hardware livre, serem criados robôs educacionais a partir de diferentes tipos de materiais e hardwares, a custos reduzidos e licenças que permitem copiar, redistribuir, adaptar, transformar e construir a partir dos mesmos. Um exemplo é a placa Arduino, criada por Barragán (2004), com nome Wiring, e disponibilizada em 2005, sob licença Creative Commons, do tipo CC BY-SA. Segundo Pezzi (2015), esses hardwares abertos e livres, e as correspondentes documentações que garantem as liberdades de uso, cópia, exploração e divulgação, constituem hiperobjetos. Estes, originados da cultura hacker, podem acrescentar qualidade à robótica educacional livre, atualmente, em 
expansão. Porém, para a construção dos robôs, são necessárias também ferramentas que possibilitem a construção de peças mecânicas ou de acabamento. É aí que entram os makerspaces educacionais, como os espaços que, através do uso de ferramentas digitais como cortadora laser e impressora 3D, tornam possíveis esses robôs.

Os makerspaces, independentemente de serem educacionais ou não, são espaços de aprendizagem, pois os projetos neles desenvolvidos são, em geral, multidisciplinares. A participação em um projeto que envolve física, engenharia, computação e design tem grandes chances de levar os sujeitos a interpretação e análise de situações e, consequentemente, a desenvolverem novos esquemas cognitivos ou, no mínimo, a melhorarem os seus esquemas conceituais pelo processo de auto-regulação.

A Robótica Educativa, com suas possibilidades de experimentação, problematização, análise de hipóteses, criação e trabalho em grupo, forma um campo rico para o aprender e a sociocognição, contrapondo o uso das metodologias passo a passo, que limitam a experiência ao que já foi pensado, testado e aprovado pelos desenvolvedores, deixando pouco desafio para provocar o pensar no sujeito. (CALLEGARI; VALENTINI, 2016, p. 111)

\section{A Abstração Reflexionante}

Embora as máquinas, ferramentas e materiais sejam importantes para o desenvolvimento de projetos de robótica, nossa observação está no processo cognitivo, nas construções cognitivas, nas abstrações, ou seja, nos processos de pensamentos reflexivos que acompanham as ações para construir algo, usando - ou não - máquinas. Para Lopes (2008), a abstração, no sentido de pensamento reflexivo, “é um processo necessário para construir noções e conceitos em relação aos fenômenos e objetos”. Essa definição é baseada nos estudos de Jean Piaget (1995), o qual classificou a abstração em dois tipos: a abstração empírica, que consiste em percepção dos aspectos físicos dos objetos ou nos aspectos materiais da própria ação do sujeito e a abstração reflexionante que se apoia sobre esses aspectos junto com as atividades cognitivas do sujeito que pratica a ação.

Enquanto o sujeito faz uso da abstração empírica, retirando as informações dos objetos usando os órgãos do sentido, a abstração reflexionante as obtém da reflexão sobre o fato. O analisar, comparar, sintetizar, repensar, refletir, reconsiderar e outras formas de pensamento reflexivo é que mudam as estruturas cognitivas. Enquanto a abstração empírica é fundamental para uma análise superficial da situação, fato ou ação, permitindo reflexionamentos sucessivos, com afirmações e contradições, a abstração reflexionante está em nível mais elevado, onde os esquemas cognitivos sofrem alterações e, consequentemente, há possibilidade de produção de conhecimento (PIAGET, 1995).

Na obra de Piaget, abstração é a atividade do sujeito conhecedor, ao mesmo tempo coordenadora e diferenciadora, mediante a qual constrói conhecimento como estrutura, competência ou capacidade utilizando características retiradas ou extraídas, ora dos objetos (abstração empírica), ora de suas coordenações de ações (abstração reflexionante - pseudoempírica ou refletida)”. (BECKER, 2017)

Para melhor compreender a abstração reflexionante, se faz necessário conhecer como ocorre a aprendizagem. Segundo Piaget (1976), a aprendizagem é uma atividade orgânica e é nos processos de assimilação, acomodação e equilibração que o 
pensamento se articula e, a partir do qual a estrutura cognitiva, frente a uma nova realidade, é organizada. Uma inquietação, dúvida, ou questionamento pode ser considerado como um desequilíbrio momentâneo ao qual o organismo tende a assimilar informações para resolver esse impasse. Junto da assimilação vem a acomodação, que pode ser com formação de novos esquemas e estruturas. A isso chamamos de equilibração, o que não consiste num equilíbrio definitivo pois novas desequilibrações e reequilibrações surgirão. "O sujeito procura evitar a incoerência e tende, pois, sempre na direção de certas formas de equilíbrio, mas sem jamais atingi-las, senão às vezes a título de etapas provisórias” (PIAGET, 1976, p. 156).

As abstrações estão presentes em todo o processo de equilibração das estruturas cognitivas. Somente ocorrem alterações dessas estruturas, ao qual Piaget (1976) chamou de equilibração majorante, quando essas se formam a partir de abstrações de alto nível, ou seja, abstrações reflexionantes. "A abstração empírica e a abstração reflexionante, estão presentes em todos os níveis de desenvolvimento, dos patamares sensório-motores até as formas mais elevadas do pensamento científico” (PIAGET, 1995). A figura 01 mostra que em todos os níveis do conhecimento, da infância até a fase do pensamento formal, a abstração empírica é sempre solidária a abstração reflexionante, sendo que esta se expande e se aprimora. O pensamento formal é, segundo Piaget (1967), caracterizado pela capacidade de separar o conteúdo da forma e, através da abstração, retirar da realidade as informações necessárias para determinar possibilidades (hipóteses) e deduzir delas as suas consequências.

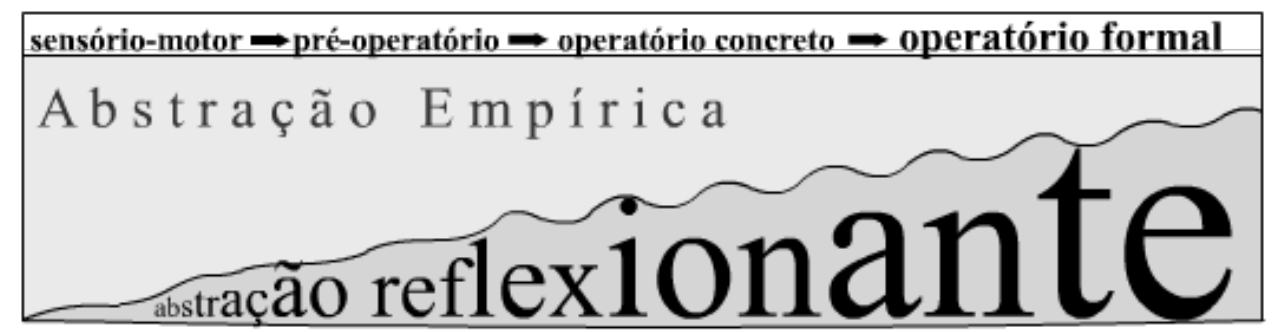

Figura 1: Relação entre as abstrações empírica e reflexionante, dos primeiros anos de vida até a idade adulta

\section{O Desenvolvimento do Pensamento Computacional e a Abstração Reflexionante}

A expressão Pensamento Computacional (PC) já havia sido usada por Papert, em 1980, para definir o tipo de pensamento que uma criança estaria desenvolvendo quando estivesse trabalhando com computadores, aos quais ele se referia "como instrumento para aprender, desenvolver a criatividade e 'concretizar' o pensamento computacional”. Mais tarde, Wing (2006) junto de instituições como a Computer Science Teachers Association (CSTA) e International Society for Technology in Education (ISTE) divulgou as vantagens de sua aplicação como processo de formulação e resolução de problemas. Uma compilação de estudos realizados na Europa, por Bocconi et al.(2016), cujo objetivo era identificar o desenvolvimento do PC e sua aplicação na escola, mostra que há pequenas divergências nestes estudos, quanto às habilidades que o envolve. Optamos em trabalhar com as habilidades do PC apresentadas a seguir:

- Coleta, análise e representação dos dados: reunião de dados de forma apropriada, análise objetiva e coerente e organização por meio de tabelas, gráficos, desenhos, esquemas, palavras ou qualquer método disponível. 
- Decomposição de problemas: divisão em problemas menores e, portanto, de mais fácil resolução.

- Algoritmos: desenvolvimento de uma série lógica e organizada de passos a serem seguidos.

- Automação: utilização de computadores/máquinas para tarefas repetitivas.

- Simulação: representação ou modelagem de um processo ou produto.

- Depuração: aplicação sistemática de pensamento lógico, testes ou rastreamento para avaliar e prever resultados.

- Paralelismo e/ou generalização: utilização do mesmo processo para resolução de uma ampla variedade de problemas semelhantes.

Alguns autores identificam a abstração como uma das habilidades do PC. Optamos em considerá-la como a habilidade pertencente ao sujeito cognoscente e, portanto, presente em todos os participantes da pesquisa e necessária para o desenvolvimento das outras sete (7) habilidades. A figura 02 mostra o PC ancorado na abstração reflexionante.

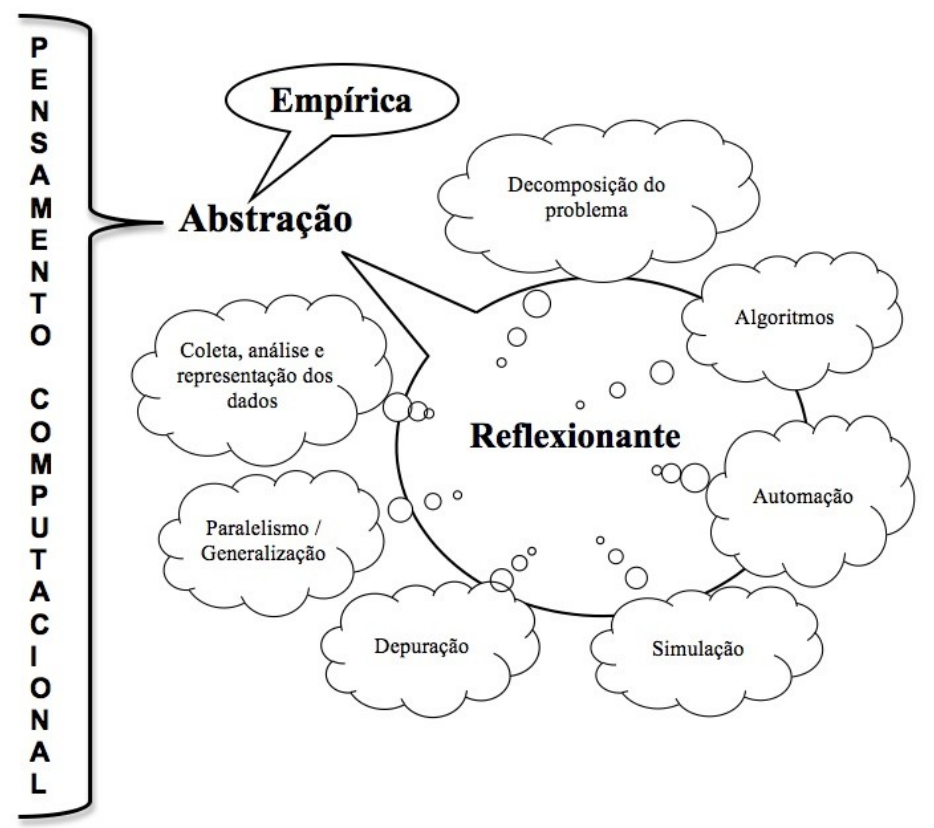

Figura 2: As abstrações empírica e reflexionante e as habilidades do pensamento computacional

Blikstein (2008), Gonzàlez (2015), Zapata-Ros (2015), Bocconi et al.(2016), Borges, Menezes \& Fagundes (2017), Lowe \& Brophy (2017), Avila \& Cavalheiro (2017) concordam que a educação pode se beneficiar se o PC for aplicado em escolas ou espaços educacionais. As vantagens apontadas são que o PC organiza e agiliza a forma de pensar nas áreas de Ciências, Tecnologia, Engenharia e Matemática (STEM Science, Technology, Engineering and Mathematics), auxilia na aprendizagem de programação e de suas linguagens de forma progressiva, favorece toda aprendizagem apoiada em tecnologia e pode contribuir para o desenvolvimento do pensamento formal.

\section{Metodologia}

O trabalho se desenvolveu na forma de pesquisa qualitativa, com a coleta de dados autorizada através de um Termo de Consentimento Livre e Esclarecido. Um grupo de 8 
alunos, de idades compreendidas entre 13 e 17 anos, que já frequentava oficinas de robótica em sua escola de ensino médio, compareceram a 12 encontros de 3 horas cada, no makerspaces POALab, do IFRS ${ }^{1}$, câmpus Porto Alegre. Estes, 5 meninos e 3 meninas, foram capacitados para o uso da cortadora a laser e da impressora 3D, para criação de imagens bi e tridimensionais e outras instruções necessárias para desenvolverem um hiperobjeto a partir de conhecimentos de robótica, com o microcontrolador Arduino e, no mínimo, um motor de corrente contínua e um sensor óptico reflexivo. O desafio proposto pode ser sintetizado no seguinte problema: “Como desenvolver um hiperobjeto mecatrônico usando a placa Arduino, um drive de motor e, no mínimo, um motor e um sensor?” Os materiais para concretização foram disponibilizados pelas pesquisadoras, mas os participantes eram livres para construir e acrescentar livremente o que desejassem.

Durante os trabalhos foi observada e analisada a conversação entre os sujeitos no grupo e fora dele, com outros grupos. Individualmente, os participantes foram submetidos a questionamentos, com a finalidade de conduzi-los a desenvolver o PC e, ao mesmo tempo, analisar a presença da abstração reflexionante nas respostas aos questionamentos feitos. Os registros dos dados foram realizados por anotações diretas, arquivos de som e/ou arquivos de vídeos.

A partir de perguntas dirigidas, referente a cada habilidade do pensamento computacional (Quadro 1), os alunos puderam refletir para responder e, dessa forma, desencadear os processos gerais de acomodação e assimilação, necessários à aprendizagem.

Quadro 1 - Perguntas elaboradas para identificar as habilidades do PC

\begin{tabular}{|c|c|}
\hline $\begin{array}{l}\text { Habilidades do } \\
\text { Pensamento } \\
\text { Computacional }\end{array}$ & Exemplos de Perguntas \\
\hline $\begin{array}{l}\text { Coleta e Análise dos } \\
\text { Dados e/ou Materiais }\end{array}$ & $\begin{array}{l}\text { - Por que coletar dados? } \\
\text { - Qual a relevância desse dado para o projeto? }\end{array}$ \\
\hline $\begin{array}{l}\text { Organização dos Dados } \\
\text { e/ou Materiais }\end{array}$ & $\begin{array}{l}\text { - Onde estão seus dados? Onde os registra? Por que registrá-los? } \\
\text { - Usar uma planilha eletrônica facilita, dificulta ou tanto faz, para } \\
\text { organizar os dados?” }\end{array}$ \\
\hline $\begin{array}{l}\text { Decomposição do } \\
\text { Problema }\end{array}$ & $\begin{array}{l}\text { - Você tem um problema que é, por exemplo, como construir um carrinho } \\
\text { seguidor de linha utilizando motor(es) e sensor(es) ópticos reflexivos? } \\
\text { Pode resolvê-lo de forma direta, levantando hipóteses, testando-as e } \\
\text { dessas ter a solução única para o seu projeto? } \\
\text { - Se a resposta foi NÃO para a pergunta anterior, o que deverá ser feito? } \\
\text { Por quê? }\end{array}$ \\
\hline Algorítmos & $\begin{array}{l}\text { - Qual a vantagem de listar uma sequência de passos de desenvolvimento } \\
\text { - } \quad \text { essa cada etapa do projeto e segui-la do início ao fim? } \\
\text { - O que você entende por algoritmo? } \\
\text { - Existe algoritmo fora do código de programação? Explique. }\end{array}$ \\
\hline Automação & $\begin{array}{l}\text { - As máquinas digitais facilitam o trabalho? Por quê? } \\
\text { - Além da programação no IDE do Arduino, que outra forma de automação } \\
\text { foi ou pode ser utilizada? }\end{array}$ \\
\hline Simulação & - Você fez simulação em que etapas do seu projeto? \\
\hline Depuração & $\begin{array}{l}\text { - Em que situação você identificou que existiam dados e/ou excedentes no } \\
\text { seu projeto? }\end{array}$ \\
\hline
\end{tabular}

1 Instituto Federal de Educação, Ciência e Tecnologia do Rio Grande do Sul 


\begin{tabular}{|c|l|}
\hline & $\bullet \quad$ Quantas vezes você refez partes do projeto? \\
\hline $\begin{array}{c}\text { Paralelismo } \\
\text { /Generalização }\end{array}$ & $\bullet \begin{array}{l}\text { Em que situações você percebeu que poderia repetir um procedimento } \\
\text { feito anteriormente, durante o desenvolvimento do projeto? Isso ocorreu } \\
\text { em que parte (Mecânica, Eletrônica ou Programação)? }\end{array}$ \\
\hline
\end{tabular}

Além de um caderno de anotações, também foram distribuídas folhas em branco tamanho A3 para os participantes. As figuras 03 e 04 mostram desenhos de dois dos projetos iniciais.

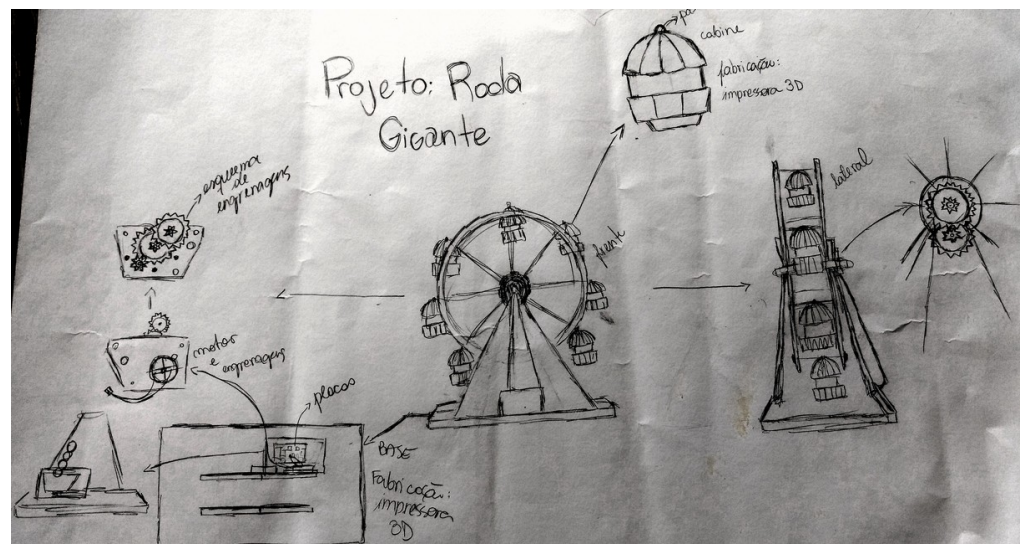

Figura 3: Projeto "Roda gigante": Etapa Inicial

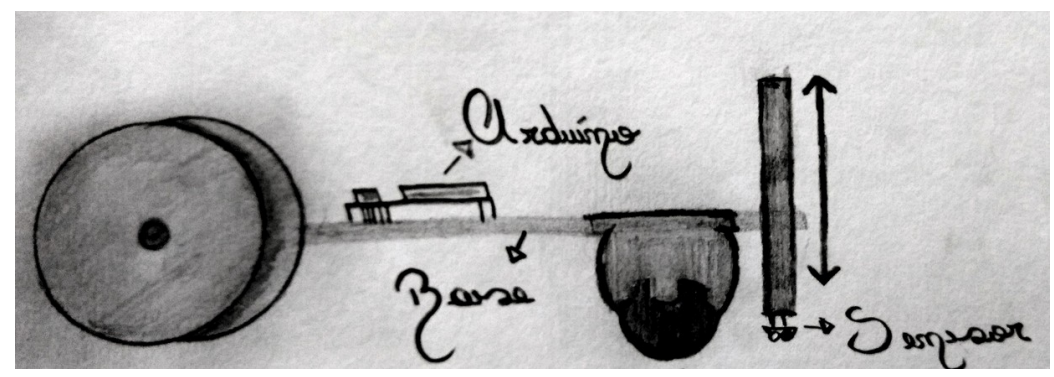

Figura 4: Projeto "O Herói”: Etapa Inicial

Dos quatro projetos desenvolvidos em duplas, e concluídos satisfatoriamente, apresentamos a foto de dois deles, em diferentes etapas. A figura 05 mostra uma simulação usando as peças reais sobre desenho com medidas reais do projeto "O Carrinho" e a figura 06 apresenta o hiperobjeto "O Herói”, já funcionando e deslocando-se sobre um faixa preta sobreposta a um fundo branco. 


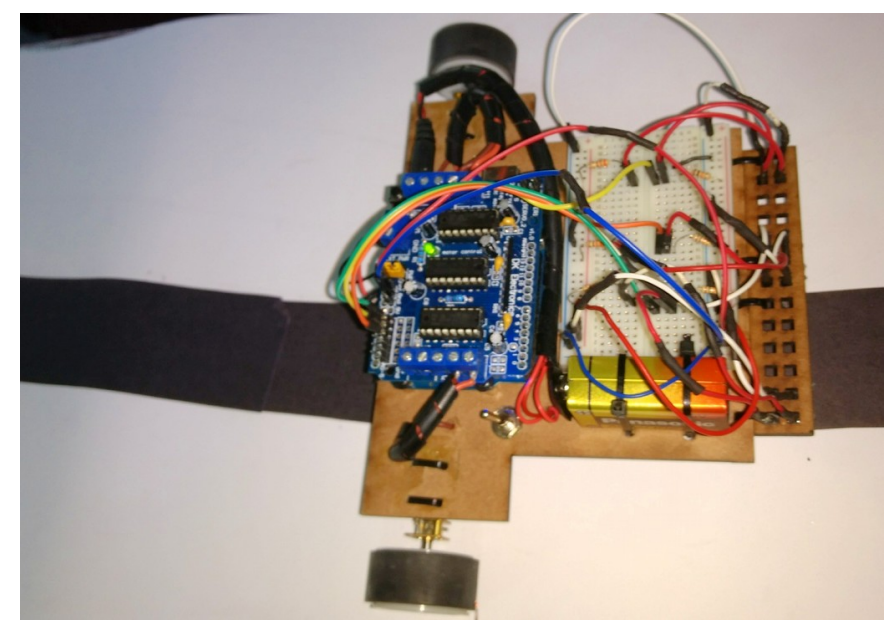

Figura 6: Projeto "O Herói" finalizado.

\section{Análise e Discussão dos Dados}

Nos parágrafos a seguir encontram-se destacados alguns exemplos de diálogos e comentários elaborados pelos alunos. Sublinhamos os termos que expressam reflexão ou questionamentos, demonstrando a presença da abstração reflexionante. Os comentários estão designados pelas letras A, B, C, D, na sequência em que foram apresentados pelo mesmo participante.

\section{a) Pesquisa e Coleta de Dados:}

O participante S2, quando perguntado sobre se havia necessidade de pesquisar e coletar dados sobre o projeto e em que instante isso deveria ser feito, forneceu duas respostas:

A: “A pesquisa pode ser feita em qualquer etapa do desenvolvimento do projeto. Ou não?”

B: "Estive pensando sobre coleta de dados para iniciar o projeto. Não é fácil! Mas entendo que temos que procurar saber o que usar e como usar os materiais antes."

Uma análise mostra que uma resposta complementa a outra. Quando o sujeito fala: "Estive pensando", "entendo", "procurar saber", "como usar" e até mesmo o "Ou não?" da primeira resposta, nos leva a crer que o sujeito se propôs a "pensar" sobre seus próprios pensamentos.

\section{b) Organização dos Dados:}

Em geral, todos os participantes apresentaram dificuldades em organizar os dados. No diálogo, com o sujeito S5 ele questionou sobre a necessidade de organizar, de alguma forma, os dados e materiais. São exemplos de comentários:

A: "Precisa registrar os dados?"

B: "Organizar os dados dá trabalho! Precisamos pensar na ordem que vamos usar, onde vamos usar esses dados, para que cada um serve."

A pesquisadora pergunta: "Mas isso não facilitará o trabalho depois?"

C: "Acho que sim. Facilita"!

A pesquisadora retoma a pergunta: "Então, porque não está sendo feito?"

D: "Porque dá trabalho. Tem que pensar nisso agora."

\section{c) Decomposição do Problema:}

Quanto a decomposição do problema, o sujeito S1 demonstra pensamento 
reflexivo quando fala:

A: "Para decompor um problema, é preciso pensar no carrinho pronto e imaginar cada parte separadamente e, ao mesmo tempo, elas se encaixando entre si. Cada parte vai ter um problema a resolver."

\section{d) Algoritmos:}

O comentário do participante S3 sobre algoritmo se divide em duas partes.

A: "A sequência de passos organiza o nosso pensamento. Eu achava que algoritmo era só o código de programação. Agora eu sei que algoritmo pode ser um roteiro bem feito!"

B: "Ao fazer um código de programação, para automatizar o movimento, estamos refletindo sobre uma situação que ainda virá a acontecer!”

\section{e) Automação:}

Quanto a automação, o exemplo de comentário escolhido foi sobre o uso da máquina cortadora a laser pelo sujeito S5, o qual explica:

A: "Tu primeiro cria a peça no Inkscape (software) e depois manda cortar.

No fazer a peça no software é que pensamos como ela será depois de cortada".

\section{f) Simulação:}

Ao ser questionado sobre porque devemos desenvolver a habilidade de simulação quando trabalhamos com robótica, o participante S5 se pronunciou da seguinte maneira:

A: "Na protoboard nós podemos errar e arrumar".

E momentos depois:

B: "A simulação é um pensamento que se coloca em prática e pode dar errado. Evitamos errar depois, simulando antes.”

\section{g) Depuração:}

S3 fez dois comentários, complementares, que são indicativos da habilidade do PC referente a depuração:

A: "No uso de jumpers (fios de conexão), em excesso, ficou um emaranhado. Tivemos que limpar e ajustar. Aplicamos depuração, né?”

B: "Tem que analisar o que se está fazendo e repensar todo ele. Se tem coisas que podem ser mudadas. Se essas mudanças vão melhorar a aparência!"

A pesquisadora pergunta: "Só a aparência?"

C: "Não! Também o funcionamento. Se questionar sobre algo que não está funcionando. Repensar outra forma."

\section{h) Paralelismo/Generalização:}

Nem todo projeto demanda as habilidades de paralelismo e generalização. No projeto de S1, identificamos o paralelismo quando o sujeito diz que repete o processo aprendido na fabricação das demais peças.

A: "Quando construímos a primeira roda, apenas repetimos o processo para as demais. Isso é aproveitar o que aprendemos, repetindo o processo."

\section{Considerações Finais}

O desafio proposto aos alunos esteve centrado em robótica educacional desenvolvida num laboratório de fabricação digital. Isso ampliou as possibilidades de ação para concretizar o projeto idealizado. Assim, vale ressaltar a importância de estruturas, 
ferramentas e mecanismos que suportam metodologias ativas de aprendizagem.

Ao analisar os diálogos e comentários, ficou evidente a presença de abstração reflexionante nas atividades de robótica e na aplicação do pensamento computacional. Conclui-se, portanto, que a abstração reflexionante faz parte de todas as habilidades do pensamento computacional e que o mesmo pode ser desenvolvido por meio de atividades práticas envolvendo robótica educacional.

O pensamento computacional tem como base a abstração, tanto empírica quanto a reflexionante. No entanto, enquanto forma de pensamento organizado e ordenado, analisando, identificando e implementando possíveis soluções para um problema, usando máquinas digitais ou não, o pensamento computacional está sendo gerido por inúmeras abstrações reflexionantes. Todas as etapas do pensamento computacional são processos de pensamento e estão sob a ação reflexiva que ora denominamos por abstração reflexionante.

Como desdobramento dessa pesquisa, identificamos a necessidade de um estudo sobre a influência de atividades de robótica educacional na abstração empírica e como essas atividades desencadeiam abstrações reflexionantes.

\section{Referências}

AHO, A. V., ULLMAN, J. D.(1992). Foundations of Computer Science. New York: W. H. Freeman and Company.

AVILA, C., CAVALHEIRO, S. (2017) Robótica Educacional como Estratégia de Promoção do Pensamento Computacional - Uma Proposta de Metodologia Baseada em Taxonomias de Aprendizagem. In: Anais dos Workshops do VI Congresso Brasileiro de Informática na Educação. Disponível em http://brie.org/pub/index.php/wcbie/article/view/7508/5303. Acesso em maio de 2018.

BARRAGÁN, H. (2004). Wiring: Prototyping Physical Interaction Design. Disponível em http://people.interactionivrea.org/h.barragan/thesis/thesis_low_res.pdf. Acesso em 20/04/2018

BECKER, F. (2017). Abstração Pseudoempírica: Significado epistemológico e impacto metodológico. Educação \& Realidade, 371-393.

BLIKSTEIN. P. (2008) O pensamento computacional e a reinvenção do computador na educação. Disponível em http://cgceducacao.com.br/o-pensamento-computacional-ereinvencao-computador-na-educacao/. Acesso em junho de 2017.

BOCCONI, S., CHIOCCARIELLO, A., DETTORI, G., FERRARI, A., ENGELHARDT, K. (2016). Developing computational thinking in compulsory education - Implications for policy and practice. Luxembourg: Publications Office of the European Union.

BORGES, K. S., MENEZES, C. S. DE, \& FAGUNDES, L. da C. (2017). The Use of Computational Thinking in Digital Fabrication Projects A Case Study From the Cognitive Perspective. In: Proceedings of Frontiers in Education - IEEE. Indianapolis, Indiana, USA.

CALLEGARI, J. H., \& VALENTINI, C. B. (2016). Robótica Educativa com crianças/jovens: processos sociocognitivos. In: Refletindo sobre educação: contribuições da história da educação, tecnologia e linguagem. Caxias do Sul, 111130 . 
GONZÀLES, M. R. (2015) Computational Thinking Test: Design Guidelines and Content Validation. In: EDULEARN15 Conference. Barcelona, 2436-2444.

LOPES, D. Q. (2008). A exploração de modelos e os níveis de abstração nas construções criativas com robótica educacional. Tese de doutorado. Informática na Educação da Universidade Federal do Rio Grande do Sul. Brasil.

LOWE, T., \& BROPHY, S. (2017). An Operationalized Model for Defining Computational Thinking. In: Proceedings of Frontiers in Education - IEEE. Indianapolis, Indiana, USA

PAPERT, S. (1980). Mindstorms: children, computers and powerful ideas. New York.

PEZZI, R. F. (2015). Ciência aberta: dos hipertextos aos hiperobjetos. In: Ciência Aberta Questões Abertas. ALBAGLI, S., MACIEL, M. L., ABDO, A. H. Organizadores. IBICT; Rio de Janeiro: UNIRIO.

PIAGET, J. (1967). O Raciocínio na criança. Rio de Janeiro. Editora Record.

PIAGET, J. (1976). A equilibração das estruturas cognitivas: problema central do desenvolvimento. Rio de Janeiro: Zahar Editores.

PIAGET, J. (1995). Abstração Reflexionante - Relações Lógico-Aritméticas e Ordem das Relações Espaciais. Porto Alegre: Artes Médicas.

WING, J. M. (2006). Computational Thinking. In Communications of the ACM, 33-35.

WING, J. M. (2014). Computational Thinking Benefits Society. Disponível em http://socialissues.cs.toronto.edu/2014/01/computational-thinking/ Acesso em março de 2017.

ZAPATA-ROS, M. (2015). Pensamiento computacional: Una nueva alfabetización digital. In Revista de Educación a Distancia. 46(4). 